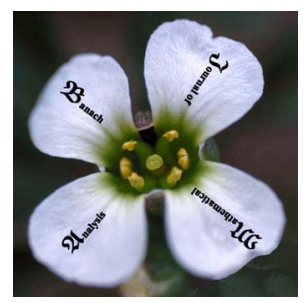

Banach J. Math. Anal. 10 (2016), no. 1, 120-132

http://dx.doi.org/10.1215/17358787-3336606

ISSN: $1735-8787$ (electronic)

http://projecteuclid.org/bjma

\title{
JESSEN-TYPE INEQUALITIES FOR SEVERAL VARIABLES VIA SUPERQUADRATIC FUNCTIONS
}

\author{
RABIA BIBI \\ Dedicated to Professor Josip Pečarić \\ Communicated by S. S. Dragomir
}

\begin{abstract}
In this paper, we give the generalizations of Jessen's inequality, Hölder's inequality, the converse Jessen inequality, and the Jessen-Mercer inequality for superquadratic functions of several variables. We also give applications of the obtained inequalities to time-scale integrals.
\end{abstract}

\section{INTRODUCTION}

Recently, the concept of superquadratic functions in one variable was introduced by S. Abramovich, G. Jameson, and G. Sinnamon in [3], and in several variables by S. Abramovich, S. Banić, and M. Matić in [1].

Let $\mathbb{R}^{m}$ be the Euclidean space, $\mathbf{x}, \mathbf{y} \in \mathbb{R}^{m}$, and let $\mathbf{f}$ be the function defined on $X \subset \mathbb{R}^{m}$. Throughout the paper, we use the following notation:

$$
\begin{aligned}
\mathbf{x} & =\left(x_{1}, x_{2}, \ldots, x_{m}\right), \quad \mathbf{y}=\left(y_{1}, y_{2}, \ldots, y_{m}\right), \\
\mathbf{x} \cdot \mathbf{y} & =\left(x_{1} y_{1}, x_{2} y_{2}, \ldots, x_{m} y_{m}\right), \quad|\mathbf{x}|=\left(\left|x_{1}\right|,\left|x_{2}\right|, \ldots,\left|x_{m}\right|\right), \\
\langle\mathbf{x}, \mathbf{y}\rangle & =\sum_{i=1}^{m} x_{i} y_{i}, \quad \nabla \mathbf{f}=\left(\partial_{1} \mathbf{f}(\mathbf{x}), \partial_{2} \mathbf{f}(\mathbf{x}), \ldots, \partial_{m} \mathbf{f}(\mathbf{x})\right) .
\end{aligned}
$$

Copyright 2016 by the Tusi Mathematical Research Group.

Received Feb. 14, 2015; Accepted Apr. 21, 2015.

2010 Mathematics Subject Classification. Primary 26D15; Secondary 26A51, 34N05.

Keywords. Jessen inequality, converse Jessen inequality, Jessen-Mercer inequality, superquadratic functions, time-scale integrals. 
Also, $\mathbf{x} \leq \mathbf{y}(\mathbf{x}<\mathbf{y})$ means that $x_{j} \leq y_{j}\left(x_{j}<y_{j}\right)$ for all $j=1,2, \ldots, m$. The null vector is denoted by $\mathbf{0}$. The subsets $K_{m}$ and $K_{m}^{+}$in $\mathbb{R}^{m}$ are defined by

$$
K_{m}=[0, \infty)^{m}=\left\{\mathbf{x} \in \mathbb{R}^{m}: \mathbf{0} \leq \mathbf{x}\right\}, \quad K_{m}^{+}=(0, \infty)^{m}=\left\{\mathbf{x} \in \mathbb{R}^{m}: \mathbf{0}<\mathbf{x}\right\} .
$$

Definition 1.1 (see [1, Definition 1]). A function $\varphi: K_{m} \rightarrow \mathbb{R}$ is said to be superquadratic if, for every $\mathbf{x} \in K_{m}$, there exists a vector $\mathbf{c}(\mathbf{x}) \in \mathbb{R}^{m}$ such that

$$
\varphi(\mathbf{y}) \geq \varphi(\mathbf{x})+\langle\mathbf{c}(\mathbf{x}), \mathbf{y}-\mathbf{x}\rangle+\varphi(|\mathbf{y}-\mathbf{x}|)
$$

holds for all $\mathbf{y} \in K_{m}$. The function $\mathbf{f}$ is said to be strictly superquadratic if (1.1) is strict for all $\mathbf{x} \neq \mathbf{y}$.

For example, the function $\varphi(\mathbf{x})=\sum_{i=1}^{m} x_{i}^{p}$ is superquadratic on $K_{m}$ for all $p \geq 2$ (see [1, Example 1]), and $\varphi(\mathbf{x})=-\left(\sum_{i=1}^{m} x_{i}^{p}\right)^{\frac{1}{p}}$ is superquadratic on $K_{m}$ for all $p \geq 1$ (see [1, Example 3]). A superquadratic function may or may not be convex. In the case of nonnegative functions, Definition 1.1 is a refinement of the definition of convex functions.

Lemma 1.2 ([1, Lemma 1]). Let $\varphi$ be a superquadratic function with $\mathbf{c}(\mathbf{x})=$ $\left(c_{1}(\mathbf{x}), c_{2}(\mathbf{x}), \ldots, c_{m}(\mathbf{x})\right)$ as in Definition 1.1. Then we have the following:

(i) $\varphi(\mathbf{0}) \leq 0$;

(ii) if $\varphi(\mathbf{0})=\nabla \varphi(\mathbf{0})=0$, then $c_{j}(\mathbf{x})=\partial_{j} \mathbf{f}(\mathbf{x})$ whenever $\partial_{j} \mathbf{f}(\mathbf{x})$ exists for some index $j=1,2, \ldots, m$, at $\mathbf{x} \in K_{m}$;

(iii) if $\varphi \geq 0$, then $\varphi$ is convex and $\varphi(\mathbf{0})=\nabla \varphi(\mathbf{0})=0$.

Jessen's inequality (1931) is a generalization of the well-known Jensen's inequality for positive linear functionals. There are many improvements and variants of Jensen's inequality and Jessen's inequality in the literature. In this paper, we give generalizations of the following Jessen-type inequalities for superquadratic functions. For this, first we give the definition of positive linear functionals.

Definition 1.3. Let $E$ be a nonempty set, and let $L$ be a linear class of real-valued functions $f: E \rightarrow \mathbb{R}$ having the following properties:

$\left(\mathrm{L}_{1}\right)$ If $f, g \in L$ and $a, b \in \mathbb{R}$, then $(a f+b g) \in L$.

$\left(\mathrm{L}_{2}\right) 1 \in L$; that is, if $f(t)=1$ for all $t \in E$, then $f \in L$.

A positive linear functional is a functional $A: L \rightarrow \mathbb{R}$ having the following properties (see [11, p. 47]):

$\left(\mathrm{A}_{1}\right)$ If $f, g \in L$ and $a, b \in \mathbb{R}$, then $A(a f+b g)=a A(f)+b A(g)$.

$\left(\mathrm{A}_{2}\right)$ If $f \in L$ and $f(t) \geq 0$ for all $t \in E$, then $A(f) \geq 0$.

If, additionally, the condition $A(1)=1$ is satisfied, then we say that $A$ is a positive normalized linear functional.

Sums and Lebesgue integrals are the most familiar examples of positive linear functionals. In [4] it is shown that time-scale integrals, including the Cauchy, Riemann, Lebesgue, multiple Riemann, multiple Lebesgue delta, nabla, and diamond- $\alpha$ time-scale integrals also satisfy the properties of positive linear functionals. 
Theorem 1.4 ([6, Theorem 10]). Let $L$ satisfy conditions $\left(L_{1}\right),\left(L_{2}\right)$, and let $A$ satisfy conditions $\left(A_{1}\right)$ and $\left(A_{2}\right)$ on a nonempty set $E$. Suppose that $w \in L$ with $w \geq 0$ and $A(w)>0$ and that $\varphi:[0, \infty) \rightarrow \mathbb{R}$ is a continuous superquadratic function. Then, for all nonnegative $f \in L$ such that $w f, w \varphi(f), w \varphi\left(\mid f-\frac{A(w f)}{A(w)}\right.$. $1 \mid) \in L$, we have

$$
\varphi\left(\frac{A(w f)}{A(w)}\right) \leq \frac{A(w \varphi(f))-A\left(w \varphi\left(\left|f-\frac{A(w f)}{A(w)} \cdot 1\right|\right)\right)}{A(w)} .
$$

Theorem 1.5 ([6, Theorem 15]). Let $L$ satisfy conditions $\left(L_{1}\right),\left(L_{2}\right)$, and let $A$ satisfy conditions $\left(A_{1}\right)$ and $\left(A_{2}\right)$ on a nonempty set $E$. Let $w \in L$ be a nonnegative function. Suppose that $\varphi:[0, \infty) \rightarrow \mathbb{R}$ is a superquadratic function. Then, for every $f \in L, f: E \rightarrow[m, M] \subseteq[0, \infty)$ such that $w f, w(\varphi \circ f) \in L$, we have

$$
A(w \varphi(f))+\Delta_{c} \leq \frac{M A(w)-A(w f)}{M-m} \varphi(m)+\frac{A(w f)-m A(w)}{M-m} \varphi(M),
$$

where

$$
\Delta_{c}=\frac{1}{M-m} A((M w-w f) \varphi(f-m \cdot 1)+(w f-m w) \varphi(M \cdot 1-f)) .
$$

Theorem 1.6 ([2, Theorem 2.3]). Let L satisfy properties $\left(L_{1}\right),\left(L_{2}\right)$ on a nonempty set $E$; let $\varphi:[0, \infty) \rightarrow \mathbb{R}$ be a continuous superquadratic function; and let $0 \leq m<M<\infty$. Assume that $A$ is an isotonic linear functional on $L$ with $A(1)=1$. If $g \in L$ is such that $m \leq g(t) \leq M$ for all $t \in E$ and such that

$$
\varphi(g), \varphi(m+M-g),(M-g) \varphi(g-m),(g-m) \varphi(M-g), \varphi(|g-A(g)|) \in L,
$$

then we have

$$
\begin{aligned}
\varphi(m & +M-A(g)) \\
\leq & \varphi(m)+\varphi(M)-A(\varphi(g)) \\
& -\frac{2}{M-m} A((g-m) \varphi(M-g)+(M-g) \varphi(g-m))-A(\varphi(|g-A(g)|)) .
\end{aligned}
$$

\section{Generalization of Jessen's inequality}

Let $L^{m}$ be the linear class of functions $\mathbf{f}: E \rightarrow \mathbb{R}^{m}$ such that

$$
\mathbf{f}(t)=\left(f_{1}(t), \ldots, f_{m}(t)\right), \quad f_{i}(t) \in L, i=1, \ldots, m .
$$

For a given linear functional $A$, we consider the linear operator $\tilde{A}=(A, \ldots, A)$ : $L^{m} \rightarrow \mathbb{R}^{m}$ defined by

$$
\tilde{A}(\mathbf{f})=\left(A\left(f_{1}\right), \ldots, A\left(f_{m}\right)\right) .
$$

If $A(1)=1$ is satisfied, then using $\left(\mathrm{A}_{1}\right)$ we also have

$$
A(\phi(\mathbf{f}))=\phi(\tilde{A}(\mathbf{f}))
$$

for every linear function $\phi$ on $\mathbb{R}^{m}$.

In the following theorem, we obtain the generalization of Jessen's inequality for superquadratic functions (Theorem 1.4). 
Theorem 2.1. Let $L$ satisfy conditions $\left(L_{1}\right)$ and $\left(L_{2}\right)$ on a nonempty set $E$; let A satisfy conditions $\left(A_{1}\right)$ and $\left(A_{2}\right)$; and let $\tilde{A}$ be defined as in (2.1). Assume that $\varphi \in \mathrm{C}\left(K_{m}, \mathbb{R}\right)$ is superquadratic and that $w \in L$ with $w \geq 0$ and $A(w)>0$. Then, for all $\mathbf{f} \in L^{m}$ such that $\mathbf{f}(E) \subset K_{m}$ and $w f_{i}, w \varphi(\mathbf{f}), w \varphi\left(\left|\mathbf{f}-\frac{\tilde{A}(w \mathbf{f})}{A(w)} \cdot 1\right|\right) \in L$, we have

$$
\varphi\left(\frac{\tilde{A}(w \mathbf{f})}{A(w)}\right) \leq \frac{A(w \varphi(\mathbf{f}))-A\left(w \varphi\left(\left|\mathbf{f}-\frac{\tilde{A}(w \mathbf{f})}{A(w)} \cdot 1\right|\right)\right)}{A(w)} .
$$

Proof. Suppose that $\varphi$ is continuous and superquadratic on $K_{m}$. Since (1.1) holds for all $\mathbf{x}, \mathbf{y} \in K_{m}$, by substituting

$$
\mathbf{x}=\frac{\tilde{A}(w \mathbf{f})}{A(w)} \quad \text { and } \quad \mathbf{y}=\mathbf{f}
$$

in (1.1), we get

$$
\varphi(\mathbf{f}) \geq \varphi\left(\frac{\tilde{A}(w \mathbf{f})}{A(w)}\right)+\left\langle\mathbf{c}\left(\frac{\tilde{A}(w \mathbf{f})}{A(w)}\right), \mathbf{f}-\frac{\tilde{A}(w \mathbf{f})}{A(w)}\right\rangle+\varphi\left(\left|\mathbf{f}-\frac{\tilde{A}(w \mathbf{f})}{A(w)}\right|\right) .
$$

Now multiplying inequality (2.4) by the function $w$ and then applying the functional $A$, we obtain

$$
\begin{aligned}
A(w \varphi(\mathbf{f})) \geq & A(w) \varphi\left(\frac{\tilde{A}(w \mathbf{f})}{A(w)}\right)+A\left(w\left\langle\mathbf{c}\left(\frac{\tilde{A}(w \mathbf{f})}{A(w)}\right), \mathbf{f}-\frac{\tilde{A}(w \mathbf{f})}{A(w)}\right\rangle\right) \\
& +A\left(w \varphi\left(\left|\mathbf{f}-\frac{\tilde{A}(w \mathbf{f})}{A(w)}\right|\right)\right) .
\end{aligned}
$$

Let

$$
\mathbf{c}\left(\frac{\tilde{A}(w \mathbf{f})}{A(w)}\right)=\left(c_{1}, \ldots, c_{m}\right) \quad \text { and } \quad \mathbf{f}=\left(f_{1}, \ldots, f_{m}\right)
$$

Then

$$
A\left(w\left\langle\mathbf{c}\left(\frac{\tilde{A}(w \mathbf{f})}{A(w)}\right), \mathbf{f}-\frac{\tilde{A}(w \mathbf{f})}{A(w)}\right\rangle\right)=A\left(w \sum_{i=1}^{n} c_{i}\left(f_{i}-\frac{A\left(w f_{i}\right)}{A(w)}\right)\right)=0 .
$$

Now by dividing (2.5) with $A(w)$, we get

$$
\frac{A(w \varphi(\mathbf{f}))}{A(w)} \geq \varphi\left(\frac{\tilde{A}(w \mathbf{f})}{A(w)}\right)+\frac{A\left(w \varphi\left(\left|\mathbf{f}-\frac{\tilde{A}(w \mathbf{f})}{A(w)}\right|\right)\right)}{A(w)}
$$

which is the required result.

Remark 2.2. In Theorem 2.1, if $\varphi$ is a nonnegative superquadratic function, then it is convex and $A(\varphi(|\mathbf{f}-A(\mathbf{f})|)) \geq 0$. In this case, we get the refinement of McShane's inequality (see [11, Theorem 2.6]):

$$
\varphi\left(\frac{\tilde{A}(w \mathbf{f})}{A(w)}\right) \leq \frac{A(w \varphi(\mathbf{f}))}{A(w)} .
$$

As a consequence of Theorem 2.1, we obtain the following generalization of Hölder's inequality for superquadratic functions. 
Corollary 2.3. Let $L$ satisfy properties $\left(L_{1}\right)$ and $\left(L_{2}\right)$ on a nonempty set $E$, and let $A$ satisfy conditions $\left(A_{1}\right)$ and $\left(A_{2}\right)$. Suppose $\mathbf{g}=\left(g_{1}, \ldots, g_{m}\right) \in L^{m}$ such that $\mathrm{g}(E) \subset K_{m}$ and $h \in L$ such that $h \geq 0$ and $A(h)>0$. If $p \geq 2$ and $q$ is defined by $\frac{1}{p}+\frac{1}{q}=1$, then

$$
\left(\sum_{i=1}^{m} A^{p}\left(h g_{i}\right)\right)^{\frac{1}{p}} \leq\left[\sum_{i=1}^{m}\left(A\left(g_{i}^{p}\right)-A\left(\left|g_{i}-h^{q-1} \frac{A\left(h g_{i}\right)}{A\left(h^{q}\right)}\right|^{p}\right)\right)\right]^{\frac{1}{p}} A^{\frac{1}{q}}\left(h^{q}\right) .
$$

Proof. Let $\varphi(\mathbf{x})=\sum_{i=1}^{m} x_{i}^{p}$, where $p \geq 2$ and $\mathbf{x}=\left(x_{1}, x_{2}, \ldots, x_{m}\right) \in K_{m}$ in Theorem 2.1. Then inequality (2.3) becomes

$$
\begin{aligned}
\sum_{i=1}^{m}\left(\frac{A\left(w f_{i}\right)}{A(w)}\right)^{p} & \leq \frac{A\left(w \sum_{i=1}^{m} f_{i}^{p}\right)-A\left(w \sum_{i=1}^{m}\left|f_{i}-\frac{A\left(w f_{i}\right)}{A(w)}\right|^{p}\right)}{A(w)} \\
& =\frac{\sum_{i=1}^{m}\left(A\left(w f_{i}^{p}\right)-A\left(w\left|f_{i}-\frac{A\left(w f_{i}\right)}{A(w)}\right|^{p}\right)\right)}{A(w)} .
\end{aligned}
$$

Now, by applying the substitution $f_{i}=h^{-\frac{q}{p}} g_{i}$ and $w=h^{q}$ in (2.7), we get

$$
\sum_{i=1}^{m}\left(\frac{A\left(h g_{i}\right)}{A\left(h^{q}\right)}\right)^{p} \leq \frac{\sum_{i=1}^{m}\left(A\left(g_{i}^{p}\right)-A\left(\left(\left|g_{i}-h^{q-1} \frac{A\left(h g_{i}\right)}{A\left(h^{q}\right)}\right|\right)^{p}\right)\right)}{A\left(h^{q}\right)} .
$$

By multiplying this inequality with $A^{p}\left(h^{q}\right)$ and then taking the power $1 / p$, we get (2.6).

Remark 2.4. If we take $m=1$ in Corollary 2.3, then inequality (2.7) is equivalent to the functional Hölder inequality given in [6, Theorem 13].

\section{Generalization of the Converse Jessen inequality}

In this section, we give the generalization of the converse Jessen inequality for superquadratic functions (Theorem 1.5) on convex hulls in $K_{m} \subset \mathbb{R}^{k}$ and analogue results for $k$-simplices in $K_{m}$. The convex hull of vectors $\mathbf{x}_{1}, \ldots, \mathbf{x}_{n} \in \mathbb{R}^{k}$ is the set

$$
\left\{\sum_{i=1}^{n} \alpha_{i} \mathbf{x}_{i} \mid \alpha_{i} \in \mathbb{R}, \alpha_{i} \geq 0, \sum_{i=1}^{n} \alpha_{i}=1\right\}
$$

and it is represented by $K=\operatorname{co}\left(\left\{\mathbf{x}_{1}, \ldots, \mathbf{x}_{n}\right\}\right)$. Barycentric coordinates over $K$ are continuous real functions $\lambda_{1}, \ldots, \lambda_{n}$ on $K$ with the following properties:

$$
\lambda_{i}(\mathbf{x}) \geq 0, i \in\{1, \ldots, n\}, \quad \sum_{i=1}^{n} \lambda_{i}(\mathbf{x})=1 \quad \text { and } \quad \mathbf{x}=\sum_{i=1}^{n} \lambda_{i}(\mathbf{x}) \mathbf{x}_{i} .
$$

If $\mathbf{x}_{2}-\mathbf{x}_{1}, \ldots, \mathbf{x}_{n}-\mathbf{x}_{1}$ are linearly independent vectors, then each $\mathbf{x} \in K$ can be written in a unique way as a convex combination of $\mathbf{x}_{1}, \ldots, \mathbf{x}_{n}$ in the form (3.1). We also consider the $k$-simplex $S=\operatorname{co}\left(\left\{\mathbf{v}_{1}, \ldots, \mathbf{v}_{k+1}\right\}\right)$ in $\mathbb{R}^{k}$, which is a convex hull of its vertices $\mathbf{v}_{1}, \ldots, \mathbf{v}_{k+1} \in \mathbb{R}^{k}$, where vertices $\mathbf{v}_{2}-\mathbf{v}_{1}, \ldots, \mathbf{v}_{k+1}-$ $\mathbf{v}_{1} \in \mathbb{R}^{k}$ are linearly independent. In this case, we denote a $k$-simplex by $S=$ 
$\left[\mathbf{v}_{1}, \ldots, \mathbf{v}_{k+1}\right]$. The barycentric coordinates $\lambda_{1}, \ldots, \lambda_{k+1}$ over $S$ are nonnegative linear polynomials that satisfy Lagrange's property:

$$
\lambda_{i}\left(\mathbf{v}_{j}\right)=\delta_{i j}= \begin{cases}1 & i=j \\ 0 & i \neq j .\end{cases}
$$

Therefore, it is known that, for each $\mathbf{x} \in S$, the barycentric coordinates $\lambda_{1}(\mathbf{x})$, $\ldots, \lambda_{k+1}(\mathbf{x})$ have the form

$$
\begin{aligned}
\lambda_{1}(\mathbf{x}) & =\frac{\operatorname{Vol}_{k}\left(\left[\mathbf{x}, \mathbf{v}_{2}, \ldots, \mathbf{v}_{k+1}\right]\right)}{\operatorname{Vol}_{k}(S)}, \\
\lambda_{2}(\mathbf{x}) & =\frac{\operatorname{Vol}_{k}\left(\left[\mathbf{v}_{1}, \mathbf{x}, \ldots, \mathbf{v}_{k+1}\right]\right)}{\operatorname{Vol}_{k}(S)}, \\
& \vdots \\
\lambda_{k+1}(\mathbf{x}) & =\frac{\operatorname{Vol}_{k}\left(\left[\mathbf{v}_{1}, \mathbf{v}_{2}, \ldots, \mathbf{v}_{k}, \mathbf{x}\right]\right)}{\operatorname{Vol}_{k}(S)},
\end{aligned}
$$

where $\mathrm{Vol}_{k}$ denotes the $k$-dimensional Lebesgue measure on $S$. Here, for example, $\left[\mathbf{v}_{1}, \mathbf{x}, \ldots, \mathbf{v}_{k+1}\right]$ denotes the subsimplex obtained by replacing $\mathbf{v}_{2}$ by $\mathbf{x}$.

In other words, we see that the barycentric coordinates $\lambda_{1}, \ldots, \lambda_{k+1}$ for each $\mathbf{x} \in S$ can be presented as the ratios of the volume of the subsimplex with one vertex in $\mathbf{x}$ and the volume of $S$.

The signed volume $\operatorname{Vol}_{k}(S)$ is given by the $(k+1) \times(k+1)$ determinant

$$
\operatorname{Vol}_{k}(S)=\frac{1}{k !}\left|\begin{array}{cccc}
1 & 1 & \ldots & 1 \\
v_{11} & v_{21} & & v_{k+1,1} \\
v_{12} & v_{22} & & v_{k+1,2} \\
\vdots & \vdots & & \vdots \\
v_{1 k} & v_{2 k} & \ldots & v_{k+1, k}
\end{array}\right|,
$$

where $\mathbf{v}_{1}=\left(v_{11}, v_{12}, \ldots, v_{1 k}\right), \ldots, \mathbf{v}_{k+1}=\left(v_{k+1,1}, v_{k+1,2}, \ldots, v_{k+1, k}\right)$. Since the vectors $\mathbf{v}_{2}-\mathbf{v}_{1}, \ldots, \mathbf{v}_{k+1}-\mathbf{v}_{1}$ are linearly independent, each $\mathbf{x} \in S$ can be written in a unique way as a convex combination of $\mathbf{v}_{1}, \ldots, \mathbf{v}_{k+1}$ in the form

$$
\begin{aligned}
\mathbf{x}= & \frac{\operatorname{Vol}_{k}\left(\left[\mathbf{x}, \mathbf{v}_{2}, \ldots, \mathbf{v}_{k+1}\right]\right)}{\operatorname{Vol}_{k}(S)} \mathbf{w}_{1}+\frac{\operatorname{Vol}_{k}\left(\left[\mathbf{v}_{1}, \mathbf{x}, \ldots, \mathbf{v}_{k+1}\right]\right)}{\operatorname{Vol}_{k}(S)} \mathbf{v}_{2} \\
& +\cdots+\frac{\operatorname{Vol}_{k}\left(\left[\mathbf{v}_{1}, \mathbf{v}_{2}, \ldots, \mathbf{v}_{k}, \mathbf{x}\right]\right)}{\operatorname{Vol}_{k}(S)} \mathbf{v}_{k+1}
\end{aligned}
$$

Theorem 3.1. Let $L$ satisfy conditions $\left(L_{1}\right)$ and $\left(L_{2}\right)$ on a nonempty set $E$, and let $A$ be a positive normalized linear functional. Let $\mathbf{x}_{1}, \ldots, \mathbf{x}_{n} \in K_{m}, K=$ $\operatorname{co}\left(\left\{\mathbf{x}_{1}, \ldots, \mathbf{x}_{n}\right\}\right)$, and $\lambda_{1}, \ldots, \lambda_{n}$ be barycentric coordinates over $K$. Assume that $\varphi \in \mathrm{C}\left(K_{m}, \mathbb{R}\right)$ is superquadratic. Then, for all $\mathbf{f} \in L^{m}$ such that $\mathbf{f}(E) \subset K$, and $\varphi(\mathbf{f}), \lambda_{i}(\mathbf{f}) \in L(i=1, \ldots, n)$, we have

$$
A(\varphi(\mathbf{f})) \leq \sum_{i=1}^{n} A\left(\lambda_{i}(\mathbf{f}) \varphi\left(\mathbf{x}_{i}\right)\right)-\sum_{i=1}^{n} A\left(\lambda_{i}(\mathbf{f}) \varphi\left(\left|\mathbf{x}_{i}-\sum_{i=1}^{n} \lambda_{i}(\mathbf{f}) \mathbf{x}_{i}\right|\right)\right) .
$$


Proof. By using the properties of barycentric coordinates, we have

$$
\lambda_{i}(\mathbf{f}(t)) \geq 0, i \in\{1, \ldots, n\}, \quad \sum_{i=1}^{n} \lambda_{i}(\mathbf{f}(t))=1 \quad \text { and } \quad \mathbf{f}(t)=\sum_{i=1}^{n} \lambda_{i}(\mathbf{f}(t)) \mathbf{x}_{i} .
$$

Suppose that $\varphi$ is superquadratic on $K_{m}$. Then

$$
\begin{aligned}
\varphi(\mathbf{f}(t)) & =\varphi\left(\sum_{i=1}^{n} \lambda_{i}(\mathbf{f}(t)) \mathbf{x}_{i}\right) \\
& \leq \sum_{i=1}^{n} \lambda_{i}(\mathbf{f}(t)) \varphi\left(\mathbf{x}_{i}\right)-\sum_{i=1}^{n} \lambda_{i}(\mathbf{f}(t)) \varphi\left(\left|\mathbf{x}_{i}-\sum_{i=1}^{n} \lambda_{i}(\mathbf{f}(t)) \mathbf{x}_{i}\right|\right) .
\end{aligned}
$$

Now, by applying the functional $A$, we get (3.2).

In next result, we give an analogue of the Theorem 3.1 for superquadratic functions defined on $k$-simplices.

Corollary 3.2. Let $L$ satisfy conditions $\left(L_{1}\right)$ and $\left(L_{2}\right)$ on a nonempty set $E$; let $A$ be a positive normalized linear functional; and let $\tilde{A}$ be defined as in (2.1). Let $S=\left[\mathbf{v}_{1}, \ldots, \mathbf{v}_{k+1}\right]$ be a k-simplex in $K_{m}$, and let $\lambda_{1}, \ldots, \lambda_{k+1}$ be barycentric coordinates over $S$. Assume that $\varphi \in \mathrm{C}(S, \mathbb{R})$ is superquadratic. Then, for all $\mathbf{f} \in L^{m}$ such that $\mathbf{f}(E) \subset S$, and $\varphi(\mathbf{f}), \lambda_{i}(\mathbf{f}) \in L(i=1, \ldots, k+1)$, we have

$$
\begin{aligned}
A(\varphi(\mathbf{f})) \leq & \sum_{i=1}^{k+1} A\left(\lambda_{i}(\mathbf{f}) \varphi\left(\mathbf{v}_{i}\right)\right)-\sum_{i=1}^{k+1} A\left(\lambda_{i}(\mathbf{f}) \varphi\left(\left|\mathbf{v}_{i}-\sum_{i=1}^{k+1} \lambda_{i}(\mathbf{f}) \mathbf{v}_{i}\right|\right)\right) \\
= & \frac{\operatorname{Vol}_{k}\left(\left[\tilde{A}(\mathbf{f}), \mathbf{v}_{2}, \ldots, \mathbf{v}_{k+1}\right]\right)}{\operatorname{Vol}_{k}(S)} \varphi\left(\mathbf{v}_{1}\right)+\cdots \\
& +\frac{\operatorname{Vol}_{k}\left(\left[\mathbf{v}_{1}, \mathbf{v}_{2}, \ldots, \mathbf{v}_{k}, \tilde{A}(\mathbf{f})\right]\right)}{\operatorname{Vol}_{k}(S)} \varphi\left(\mathbf{v}_{k+1}\right) \\
& -\sum_{i=1}^{k+1} A\left(\lambda_{i}(\mathbf{f}) \varphi\left(\left|\mathbf{v}_{i}-\sum_{i=1}^{k+1} \lambda_{i}(\mathbf{f}) \mathbf{v}_{i}\right|\right)\right) .
\end{aligned}
$$

Proof. The proof is analogous to the proof of Theorem 3.1 with

$$
\lambda_{1}(\mathbf{f}(t))=\frac{\operatorname{Vol}_{k}\left(\left[\mathbf{f}(t), \mathbf{v}_{2}, \ldots, \mathbf{v}_{k+1}\right]\right)}{\operatorname{Vol}_{k}(S)}=\frac{\frac{1}{k !}\left|\begin{array}{cccc}
1 & 1 & \ldots & 1 \\
f_{1}(t) & v_{21} & & v_{k+1,1} \\
\vdots & \vdots & & \vdots \\
f_{k}(t) & v_{2 k} & \ldots & v_{k+1, k}
\end{array}\right|}{\frac{1}{k !}\left|\begin{array}{cccc}
1 & 1 & \ldots & 1 \\
v_{11} & v_{21} & v_{k+1,1} \\
v_{12} & v_{22} & v_{k+1,2} \\
\vdots & \vdots & & \vdots \\
v_{1 k} & v_{2 k} & \ldots & v_{k+1, k}
\end{array}\right|}
$$




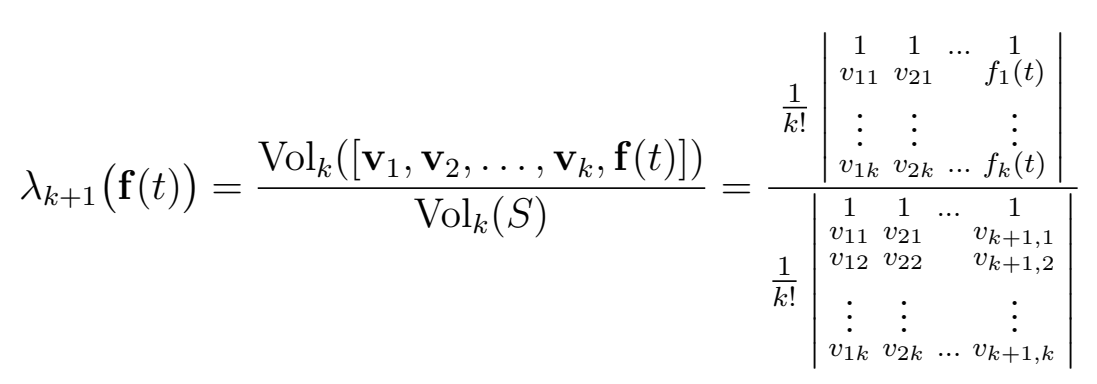

and

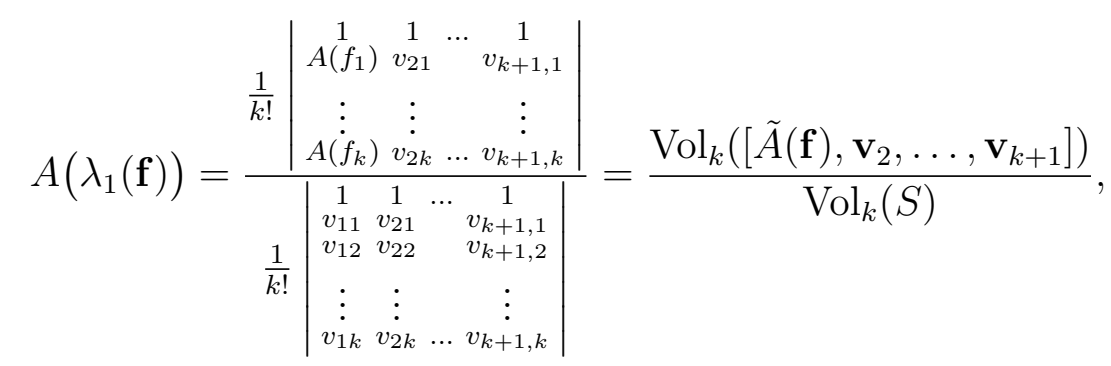

$$
\begin{aligned}
& \vdots
\end{aligned}
$$

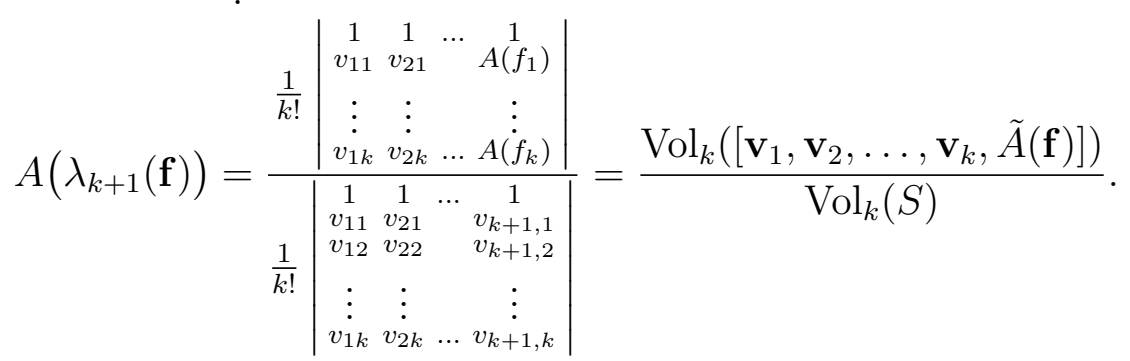

Remark 3.3. Let $f \in L$ be such that $f(E) \subset I=[m, M]$. Since the interval $I$ is a 1-simplex with vertices $m$ and $M$, the barycentric coordinates have the special form

$$
\lambda_{1}(f(t))=\frac{M-f(t)}{M-m} \quad \text { and } \quad \lambda_{2}(f(t))=\frac{f(t)-m}{M-m} .
$$

In this case, Corollary 3.2 is equivalent to Theorem 1.5.

\section{Generalization of the Jessen-Mercer inequality}

In this section, we give the generalization of the Jessen-Mercer inequality for superquadratic functions (Theorem 1.6) on convex hulls in $K_{m} \subset \mathbb{R}^{k}$ and analogue results for $k$-simplices in $K_{m} \subset \mathbb{R}^{k}$.

Theorem 4.1. Let $L$ satisfy conditions $\left(L_{1}\right)$ and $\left(L_{2}\right)$ on a nonempty set $E$; let $A$ be a positive normalized linear functional; and let $\tilde{A}$ be defined as in (2.1). Let $\mathbf{x}_{1}, \ldots, \mathbf{x}_{n} \in K_{m}, K=\operatorname{co}\left(\left\{\mathbf{x}_{1}, \ldots, \mathbf{x}_{n}\right\}\right)$, and let $\lambda_{1}, \ldots, \lambda_{n}$ be barycentric coordinates over $K$. Suppose that $\varphi \in \mathrm{C}\left(K_{m}, \mathbb{R}\right)$ is superquadratic and $\mathbf{f} \in L^{m}$ such that $\mathbf{f}(E) \subset K$ and $\varphi(\mathbf{f}), \lambda_{i}(\mathbf{f}) \in L(i=1, \ldots, n)$. If $p_{1}, \ldots, p_{n}$ are positive real numbers with $P_{n}=\sum_{i=1}^{n} p_{i}$, satisfying the condition

$$
p_{i} \geq 1 \text { for all } i=1, \ldots, n,
$$


then we have

$$
\begin{aligned}
\varphi\left(\frac{\sum_{i=1}^{n} p_{i} \mathbf{x}_{i}-\tilde{A}(\mathbf{f})}{P_{n}-1}\right) \leq & \frac{\sum_{i=1}^{n} p_{i} \varphi\left(\mathbf{x}_{i}\right)-\sum_{i=1}^{n} A\left(\lambda_{i}(\mathbf{f})\right) \varphi\left(\mathbf{x}_{i}\right)}{P_{n}-1} \\
& -\frac{\sum_{i=1}^{n}\left(p_{i}-A\left(\lambda_{i}(\mathbf{f})\right) \varphi\left(\left|\mathbf{x}_{i}-\frac{\sum_{i=1}^{n} p_{i} \mathbf{x}_{i}-\tilde{A}(\mathbf{f})}{P_{n}-1}\right|\right)\right.}{P_{n}-1} .
\end{aligned}
$$

Proof. By using the properties of barycentric coordinates, we have $\lambda_{i}(\mathbf{f}(t)) \geq 0, i \in\{1, \ldots, n\}, \quad \sum_{i=1}^{n} \lambda_{i}(\mathbf{f}(t))=1 \quad$ and $\quad \mathbf{f}(t)=\sum_{i=1}^{n} \lambda_{i}(\mathbf{f}(t)) \mathbf{x}_{i}$.

Suppose that $\varphi$ is superquadratic on $K_{m}$. Then

$$
\begin{aligned}
\varphi(\mathbf{f}(t)) & =\varphi\left(\sum_{i=1}^{n} \lambda_{i}(\mathbf{f}(t)) \mathbf{x}_{i}\right) \\
& \leq \sum_{i=1}^{n} \lambda_{i}(\mathbf{f}(t)) \varphi\left(\mathbf{x}_{i}\right)-\sum_{i=1}^{n} \lambda_{i}(\mathbf{f}(t)) \varphi\left(\left|\mathbf{x}_{i}-\sum_{i=1}^{n} \lambda_{i}(\mathbf{f}(t)) \mathbf{x}_{i}\right|\right) .
\end{aligned}
$$

Now, by applying the functional $A$, we get

$$
\begin{aligned}
A(\varphi(\mathbf{f})) \leq & \sum_{i=1}^{n} A\left(\lambda_{i}(\mathbf{f}(t))\right) \varphi\left(\mathbf{x}_{i}\right) \\
& -\sum_{i=1}^{n} A\left(\lambda_{i}(\mathbf{f}(t)) \varphi\left(\left|\mathbf{x}_{i}-\sum_{i=1}^{n} \lambda_{i}(\mathbf{f}(t)) \mathbf{x}_{i}\right|\right)\right),
\end{aligned}
$$

where

$$
\sum_{i=1}^{n} A\left(\lambda_{i}(\mathbf{f}(t))\right)=A\left(\sum_{i=1}^{n} \lambda_{i}(\mathbf{f}(t))\right)=1 \quad \text { and } \quad A\left(\lambda_{i}(\mathbf{f}(t))\right) \leq 1 .
$$

Also, we have

$$
\tilde{A}(\mathbf{f}(t))=\sum_{i=1}^{n} A\left(\lambda_{i}(\mathbf{f}(t))\right) \mathbf{x}_{i}
$$

Now we can write

$$
\begin{aligned}
\frac{\sum_{i=1}^{n} p_{i} \mathbf{x}_{i}-\tilde{A}(\mathbf{f})}{P_{n}-1} & =\frac{\sum_{i=1}^{n} p_{i} \mathbf{x}_{i}-\sum_{i=1}^{n} A\left(\lambda_{i}(\mathbf{f}(t))\right) \mathbf{x}_{i}}{P_{n}-1} \\
& =\frac{\sum_{i=1}^{n}\left(p_{i}-A\left(\lambda_{i}(\mathbf{f}(t))\right)\right) \mathbf{x}_{i}}{P_{n}-1}
\end{aligned}
$$

Since

$$
\frac{\sum_{i=1}^{n}\left(p_{i}-A\left(\lambda_{i}(\mathbf{f}(t))\right)\right)}{P_{n}-1}=1 \quad \text { and } \quad p_{i} \geq 1 \geq A\left(\lambda_{i}(\mathbf{f}(t))\right),
$$

the expression

$$
\frac{\sum_{i=1}^{n}\left(p_{i}-A\left(\lambda_{i}(\mathbf{f}(t))\right)\right)}{P_{n}-1}
$$


is a convex combination of $\mathbf{x}_{1}, \ldots, \mathbf{x}_{n}$ and belongs to $K$. Since $\varphi$ is superquadratic on $K$, we have

$$
\begin{aligned}
\varphi\left(\frac{\sum_{i=1}^{n} p_{i} \mathbf{x}_{i}-\tilde{A}(\mathbf{f})}{P_{n}-1}\right)= & \varphi\left(\frac{\sum_{i=1}^{n}\left(p_{i}-A\left(\lambda_{i}(\mathbf{f}(t))\right)\right) \mathbf{x}_{i}}{P_{n}-1}\right) \\
\leq & \frac{\sum_{i=1}^{n}\left(p_{i}-A\left(\lambda_{i}(\mathbf{f}(t))\right)\right) \varphi\left(\mathbf{x}_{i}\right)}{P_{n}-1} \\
& -\frac{\sum_{i=1}^{n}\left(p_{i}-A\left(\lambda_{i}(\mathbf{f}(t))\right)\right) \varphi\left(\left|\mathbf{x}_{i}-\frac{\sum_{i=1}^{n} p_{i} \mathbf{x}_{i}-\tilde{A}(\mathbf{f})}{P_{n}-1}\right|\right)}{P_{n}-1} .
\end{aligned}
$$

Corollary 4.2. Let $L$ satisfy conditions $\left(L_{1}\right)$ and $\left(L_{2}\right)$ on a nonempty set $E$; let $A$ be a positive normalized linear functional; and let $\tilde{A}$ be defined as in (2.1). Let $S=\left[\mathbf{v}_{1}, \ldots, \mathbf{v}_{k+1}\right]$ be a k-simplex in $K_{m}$, and let $\lambda_{1}, \ldots, \lambda_{k+1}$ be barycentric coordinates over $S$. Assume that $\varphi \in \mathrm{C}(S, \mathbb{R})$ is superquadratic. Then, for all $\mathbf{f} \in L^{m}$ such that $\mathbf{f}(E) \subset K$ and $\varphi(\mathbf{f}), \lambda_{i}(\mathbf{f}) \in L(i=1, \ldots, k+1)$, we have

$$
\begin{aligned}
\varphi( & \left.\frac{(k+1) \sum_{i=1}^{k+1} \mathbf{v}_{i}-\tilde{A}(\mathbf{f})}{k}\right) \\
\leq & \frac{(k+1) \sum_{i=1}^{k+1} \varphi\left(\mathbf{v}_{i}\right)-\sum_{i=1}^{k+1} A\left(\lambda_{i}(\mathbf{f})\right) \varphi\left(\mathbf{x}_{i}\right)}{k} \\
& -\frac{\sum_{i=1}^{k+1}\left(1-A\left(\lambda_{i}(\mathbf{f}(t))\right)\right) \varphi\left(\left|\mathbf{v}_{i}-\frac{\sum_{i=1}^{k+1} \mathbf{v}_{i}-\tilde{A}(\mathbf{f})}{k}\right|\right)}{k} .
\end{aligned}
$$

Proof. By considering $\mathbf{x}_{i}=\mathbf{v}_{i}$ and $p_{i}=1, i=1, \ldots, k+1$, in Theorem 4.1, inequality (4.7) easily follows from (4.1).

\section{Applications on time-SCAlE integrals}

A time scale $\mathbb{T}$ is an arbitrary closed subset of $\mathbb{R}$. Time-scale calculus provides unification and extension of discrete and continuous analysis. For example, when $\mathbb{T}=\mathbb{R}$, the time-scale integral is an ordinary integral, and when $\mathbb{T}=\mathbb{Z}$, the time-scale integral becomes a sum. Here, we give a brief introduction of time-scale integrals; for a detailed introduction we refer the reader to [7], [8], [9] and [10]. In [8], the multiple Lebesgue delta integral is defined in the following way.

Let $\mathbb{T}_{i}, i=1, \ldots, n$, be time scales, and let

$$
\Lambda^{n}=\mathbb{T}_{1} \times \cdots \times \mathbb{T}_{n}=\left\{t=\left(t_{1}, \ldots, t_{n}\right): t_{i} \in \mathbb{T}_{i}, 1 \leq i \leq n\right\}
$$

be an $n$-dimensional time scale. Let $f: E \rightarrow \mathbb{R}$ be a $\Delta$-measurable function, where $E \subset \Lambda^{n}$ is $\Delta$-measurable. Then the corresponding $\Delta$-integral, called the Lebesgue $\Delta$-integral, is denoted by

$$
\begin{aligned}
& \int_{E} f\left(t_{1}, \ldots, t_{n}\right) \Delta_{1} t_{1} \cdots \Delta_{n} t_{n}, \quad \int_{E} f(t) \Delta t, \\
& \int_{E} f \mathrm{~d} \mu_{\Delta}, \quad \text { or } \quad \int_{E} f(t) \mathrm{d} \mu_{\Delta}(t),
\end{aligned}
$$

where $\mu_{\Delta}$ is a $\sigma$-additive Lebesgue $\Delta$-measure on $\Lambda^{n}$. By [8, Section 3], all theorems of the general Lebesgue integration theory hold also for the Lebesgue 
$\Delta$-integral on $\Lambda^{n}$. Throughout this section, we consider $E$ to be a $\Delta$-measurable subset of $\Lambda^{n}$. Also, if $\mathbf{f}(t)=\left(f_{1}(t), \ldots, f_{n}(t)\right)$ is an $n$-tuple of functions such that the $f_{1}, \ldots, f_{n}$ are Lebesgue $\Delta$-integrable on $E$, then $\int_{E} \mathbf{f} \mathrm{d} \mu_{\Delta}$ denotes the $n$-tuple

$$
\left(\int_{E} f_{1} \mathrm{~d} \mu_{\Delta}, \ldots, \int_{E} f_{n} \mathrm{~d} \mu_{\Delta}\right)
$$

that is, the $\Delta$-integral acts on each component of $\mathbf{f}$.

The following results easily follow from the results given in Sections 2, 3, and 4, respectively, by using the fact that the multiple Lebesgue $\Delta$-integral is a positive linear functional.

Corollary 5.1. Assume that $\varphi \in \mathrm{C}\left(K_{m}, \mathbb{R}\right)$ is superquadratic and that $w$ is $a \Delta$-integrable function such that $w \geq 0$ and $\int_{E} w \mathrm{~d} \mu_{\Delta}>0$. Then, for all $\Delta$-integrable functions $\mathbf{f}$ such that $\mathbf{f}(E) \subset K_{m}$ and $w f_{i}, w \varphi(\mathbf{f})$ are $\Delta$-integrable, we have

$$
\varphi\left(\frac{\int_{E} w \mathbf{f} \mathrm{d} \mu_{\Delta}}{\int_{E} w \mathrm{~d} \mu_{\Delta}}\right) \leq \frac{\int_{E} w \varphi(\mathbf{f}) \mathrm{d} \mu_{\Delta}-\int_{E} w \varphi\left(\left|\mathbf{f}-\frac{\int_{E} w \mathbf{f} \mathrm{d} \mu_{\Delta}}{\int_{E} w \mathrm{~d} \mu_{\Delta}} \cdot 1\right|\right) \mathrm{d} \mu_{\Delta}}{\int_{E} w \mathrm{~d} \mu_{\Delta}} .
$$

Remark 5.2. Corollary 5.1 gives a generalization of Jensen's inequality on time scales for superquadratic functions (see [5, Theorem 2.5]).

Corollary 5.3. Let $g_{i}, i=1, \ldots, m$ be $\Delta$-integrable, and let $\mathbf{g}=\left(g_{1}, \ldots, g_{m}\right)$ such that $\mathbf{g}(E) \subset K_{m}$. Let $h$ be $\Delta$-integrable such that $h \geq 0$ and $\int_{E} h \mathrm{~d} \mu_{\Delta}>0$. If $p \geq 2$ and $q$ is defined by $\frac{1}{p}+\frac{1}{q}=1$, then

$$
\begin{aligned}
& \left(\sum_{i=1}^{m}\left(\int_{E} h g_{i} \mathrm{~d} \mu_{\Delta}\right)^{p}\right)^{\frac{1}{p}} \\
& \quad \leq\left[\sum_{i=1}^{m}\left(\int_{E} g_{i}^{p} \mathrm{~d} \mu_{\Delta}-\int_{E}\left|g_{i}-h^{q-1} \frac{A\left(h g_{i}\right)}{A\left(h^{q}\right)}\right|^{p} \mathrm{~d} \mu_{\Delta}\right)\right]^{\frac{1}{p}}\left(\int_{E} h^{q} \mathrm{~d} \mu_{\Delta}\right)^{\frac{1}{q}} .
\end{aligned}
$$

Remark 5.4. Corollary 5.3 gives a generalization of Hölder's inequality on time scales for superquadratic functions (see [5, Theorem 3.2]).

Corollary 5.5. Let $\mathbf{x}_{1}, \ldots, \mathbf{x}_{n} \in K_{m}$; let $K=\operatorname{co}\left(\left\{\mathbf{x}_{1}, \ldots, \mathbf{x}_{n}\right\}\right)$; let $\lambda_{1}, \ldots, \lambda_{n}$ be barycentric coordinates over $K$; and let $\int_{E} 1 \mathrm{~d} \mu_{\Delta}=1$. Assume that $\varphi \in \mathrm{C}\left(K_{m}, \mathbb{R}\right)$ is superquadratic. Then, for all $\Delta$-integrable functions $\mathbf{f}$ such that $\mathbf{f}(E) \subset K$, and $\varphi(\mathbf{f}), \lambda_{i}(\mathbf{f})(i=1, \ldots, n) \Delta$-integrable, we have

$$
\begin{aligned}
\int_{E} \varphi(\mathbf{f}) \mathrm{d} \mu_{\Delta} \leq & \sum_{i=1}^{n} \int_{E} \lambda_{i}(\mathbf{f}) \varphi\left(\mathbf{x}_{i}\right) \mathrm{d} \mu_{\Delta} \\
& -\sum_{i=1}^{n} \int_{E} \lambda_{i}(\mathbf{f}) \varphi\left(\left|\mathbf{x}_{i}-\sum_{i=1}^{n} \lambda_{i}(\mathbf{f}) \mathbf{x}_{i}\right|\right) \mathrm{d} \mu_{\Delta} .
\end{aligned}
$$

Corollary 5.6. Let $S=\left[\mathbf{v}_{1}, \ldots, \mathbf{v}_{k+1}\right]$ be a $k$-simplex in $K_{m}$; let $\lambda_{1}, \ldots, \lambda_{k+1}$ be barycentric coordinates over $S$; and let $\int_{E} 1 \mathrm{~d} \mu_{\Delta}=1$. Assume that $\varphi \in \mathrm{C}(S, \mathbb{R})$ 
is superquadratic. Then, for all $\Delta$-integrable functions $\mathbf{f}$ such that $\mathbf{f}(E) \subset S$, and $\varphi(\mathbf{f}), \lambda_{i}(\mathbf{f})(i=1, \ldots, k+1) \Delta$-integrable, we have

$$
\begin{aligned}
\int_{E} \varphi(\mathbf{f}) \mathrm{d} \mu_{\Delta} \leq & \sum_{i=1}^{k+1} \int_{E} \lambda_{i}(\mathbf{f}) \varphi\left(\mathbf{v}_{i}\right) \mathrm{d} \mu_{\Delta}-\sum_{i=1}^{k+1} \int_{E} \lambda_{i}(\mathbf{f}) \varphi\left(\left|\mathbf{v}_{i}-\sum_{i=1}^{k+1} \lambda_{i}(\mathbf{f}) \mathbf{v}_{i}\right|\right) \mathrm{d} \mu_{\Delta} \\
= & \frac{\operatorname{Vol}_{k}\left(\left[\int_{E} \mathbf{f} \mathrm{d} \mu_{\Delta}, \mathbf{v}_{2}, \ldots, \mathbf{v}_{k+1}\right]\right)}{\operatorname{Vol}_{k}(S)} \varphi\left(\mathbf{v}_{1}\right)+\cdots \\
& +\frac{\operatorname{Vol}_{k}\left(\left[\mathbf{v}_{1}, \mathbf{v}_{2}, \ldots, \mathbf{v}_{k}, \int_{E} \mathbf{f} \mathrm{d} \mu_{\Delta}\right]\right)}{\operatorname{Vol}_{k}(S)} \varphi\left(\mathbf{v}_{k+1}\right) \\
& -\sum_{i=1}^{k+1} \int_{E} \lambda_{i}(\mathbf{f}) \varphi\left(\left|\mathbf{v}_{i}-\sum_{i=1}^{k+1} \lambda_{i}(\mathbf{f}) \mathbf{v}_{i}\right|\right) \mathrm{d} \mu_{\Delta} .
\end{aligned}
$$

Remark 5.7. Corollaries 5.5 and 5.6, gives generalizations of the converse Jensen inequality on time scales for superquadratic functions (see [5, Theorem 6.2]).

Corollary 5.8. Let $\mathbf{x}_{1}, \ldots, \mathbf{x}_{n} \in K_{m}$; let $K=\operatorname{co}\left(\left\{\mathbf{x}_{1}, \ldots, \mathbf{x}_{n}\right\}\right)$; let $\lambda_{1}, \ldots, \lambda_{n}$ be barycentric coordinates over $K$; and let $\int_{E} 1 \mathrm{~d} \mu_{\Delta}=1$. Suppose that $\varphi \in \mathrm{C}\left(K_{m}, \mathbb{R}\right)$ is superquadratic and that $\mathbf{f}$ is $\Delta$-integrable such that $\mathbf{f}(E) \subset K$ and $\varphi(\mathbf{f}), \lambda_{i}(\mathbf{f})$ $(i=1, \ldots, n)$ are $\Delta$-integrable. If $p_{1}, \ldots, p_{n}$ are positive real numbers with $P_{n}=$ $\sum_{i=1}^{n} p_{i}$, satisfying the condition

$$
p_{i} \geq 1 \quad \text { for all } i=1, \ldots, n,
$$

then we have

$$
\begin{aligned}
\varphi( & \left.\frac{\sum_{i=1}^{n} p_{i} \mathbf{x}_{i}-\int_{E} \mathbf{f} \mathrm{d} \mu_{\Delta}}{P_{n}-1}\right) \\
& \leq \frac{\sum_{i=1}^{n} p_{i} \varphi\left(\mathbf{x}_{i}\right)-\sum_{i=1}^{n} \int_{E} \lambda_{i}(\mathbf{f}) \mathrm{d} \mu_{\Delta} \varphi\left(\mathbf{x}_{i}\right)}{P_{n}-1} \\
& -\frac{\sum_{i=1}^{n}\left(p_{i}-\int_{E} \lambda_{i}\left(\mathbf{f}(t) \mathrm{d} \mu_{\Delta}\right) \varphi\left(\left|\mathbf{x}_{i}-\frac{\sum_{i=1}^{n} p_{i} \mathbf{x}_{i}-\int_{E} \mathbf{f} \mathrm{d} \mu_{\Delta}}{P_{n}-1}\right|\right)\right.}{P_{n}-1} .
\end{aligned}
$$

Corollary 5.9. Let $S=\left[\mathbf{v}_{1}, \ldots, \mathbf{v}_{k+1}\right]$ be a $k$-simplex in $K_{m}$; let $\lambda_{1}, \ldots, \lambda_{k+1}$ be barycentric coordinates over $S$; and let $\int_{E} 1 \mathrm{~d} \mu_{\Delta}=1$. Assume that $\varphi \in \mathrm{C}(S, \mathbb{R})$ is superquadratic. Then, for all $\Delta$-integrable $\mathbf{f}$ such that $\mathbf{f}(E) \subset K$ and $\varphi(\mathbf{f}), \lambda_{i}(\mathbf{f}) \in$ $L(i=1, \ldots, k+1)$, we have

$$
\begin{aligned}
\varphi( & \left.\frac{(k+1) \sum_{i=1}^{k+1} \mathbf{v}_{i}-\int_{E} \mathbf{f} \mathrm{d} \mu_{\Delta}}{k}\right) \\
\leq & \frac{(k+1) \sum_{i=1}^{k+1} \varphi\left(\mathbf{v}_{i}\right)-\sum_{i=1}^{k+1} \int_{E} \lambda_{i}(\mathbf{f}) \mathrm{d} \mu_{\Delta} \varphi\left(\mathbf{x}_{i}\right)}{k} \\
& -\frac{\sum_{i=1}^{k+1}\left(1-\int_{E} \lambda_{i}\left(\mathbf{f}(t) \mathrm{d} \mu_{\Delta}\right) \varphi\left(\left|\mathbf{v}_{i}-\frac{\sum_{i=1}^{k+1} \mathbf{v}_{i}-\int_{E} \mathbf{f} \mathrm{d} \mu_{\Delta}}{k}\right|\right)\right.}{k} .
\end{aligned}
$$

Remark 5.10. Corollaries 5.8 and 5.9, gives generalizations of the Jensen-Mercer inequality on time scales for superquadratic functions (see [5, Theorem 5.2]). 
Remark 5.11. The above corollaries also hold for many other time-scale integrals, such as Cauchy, Riemann, Lebesgue, multiple Riemann, multiple Lebesgue delta, nabla, and diamond- $\alpha$ time-scale integrals, as we know that these integrals are isotonic linear functionals.

\section{REFERENCES}

1. S. Abramovich, S. Banić, and M. Matić, Superquadratic functions in several variables, J. Math. Anal. Appl. 327 (2007), no. 2, 1444-1460. Zbl 1120.26005. MR2281651. DOI 10.1016/j.jmaa.2006.05.014. 120, 121

2. S. Abramovich, J. Barić, and J. Pečarić, A variant of Jessen's inequality of Mercer's type for superquadratic functions, J. Inequal. Pure Appl. Math. 9 (2008), no. 3, 1-13. MR2443737. 122

3. S. Abramovich, G. Jameson, and G. Sinnamon, Refining Jensen's inequality, Bull. Math. Soc. Sci. Math. Roumanie (N.S.) 47(95) (2004), nos. 1-2, 3-14. MR2116376. 120

4. M. Anwar, R. Bibi, M. Bohner, and J. Pečarić, Integral inequalities on time scales via the theory of isotonic linear functionals, Abstr. Appl. Anal. 2011 (2011), art. ID 483595. Zbl 1221.26026. MR2819769. DOI 10.1155/2011/483595. 121

5. J. Barić, R. Bibi, M. Bohner, and J. Pečarić, Time scales integral inequalities for superquadratic functions, J. Korean Math. Soc. 50 (2013), no. 3, 465-477. Zbl 1279.26068. MR3056916. DOI 10.4134/JKMS.2013.50.3.465. 130, 131

6. S. Banić and S. Varošanec, Functional inequalities for superquadratic functions, Int. J. Pure Appl. Math. 43 (2008), no. 4, 537-549. MR2396478. 122, 124

7. M. Bohner and G. S. Guseinov, Multiple integration on time scales, Dynam. Systems Appl. 14 (2005), nos. 3-4, 579-606. Zbl 1095.26006. MR2179167. 129

8. M. Bohner and G. S. Guseinov, Multiple Lebesgue integration on time scales, Adv. Difference Equ. 2006 (2006), art. ID 26391. Zbl 1139.39023. MR2255162. 129

9. M. Bohner and A. Peterson, Dynamic Equations on Time Scales: An Introduction with Applications, Birkhäuser, Boston, 2001. Zbl 0978.39001. MR1843232. DOI 10.1007/978-1-4612-0201-1. 129

10. M. Bohner and A. Peterson, Advances in Dynamic Equations on Time Scales: An Introduction with Applications, Birkhäuser, Boston, 2003. Zbl 1025.34001. MR1962542. DOI 10.1007/978-0-8176-8230-9. 129

11. J. E. Pečarić, F. Proschan, and Y. L. Tong, Convex Functions, Partial Orderings, and Statistical Applications, Math. Sci. Engrg. 187, Academic Press, Boston, 1992. MR1162312. 121,123

Department of Mathematics, Hazara University, Mansehra, KPK, Pakistan.

E-mail address: emaorr@gmail.com 